\title{
Global Robust Exponential Stability and Periodic Solutions for Interval Cohen-Grossberg Neural Networks with Mixed Delays
}

\author{
Yanke Du and Rui Xu \\ Institute of Applied Mathematics, Shijiazhuang Mechanical Engineering College, Shijiazhuang 050003, China \\ Correspondence should be addressed to Yanke Du; yankedu2011@163.com
}

Received 28 June 2013; Accepted 30 September 2013

Academic Editor: Xiang Ping Yan

Copyright (C) 2013 Y. Du and R. Xu. This is an open access article distributed under the Creative Commons Attribution License, which permits unrestricted use, distribution, and reproduction in any medium, provided the original work is properly cited.

A class of interval Cohen-Grossberg neural networks with time-varying delays and infinite distributed delays is investigated. By employing H-matrix and M-matrix theory, homeomorphism techniques, Lyapunov functional method, and linear matrix inequality approach, sufficient conditions are established for the existence, uniqueness, and global robust exponential stability of the equilibrium point and the periodic solution to the neural networks. Our results improve some previously published ones. Finally, numerical examples are given to illustrate the feasibility of the theoretical results and further to exhibit that there is a characteristic sequence of bifurcations leading to a chaotic dynamics, which implies that the system admits rich and complex dynamics.

\section{Introduction}

In the past two decades, neural networks have received a great deal of attention due to the extensive applications in many areas such as signal processing, associative memory, pattern recognition, and parallel computation and optimization. It should be pointed out that the successful applications heavily rely on the dynamic behaviors of neural networks. Stability, as one of the most important properties of neural networks, is crucially required when designing neural networks.

In electronic implementation of neural networks, there exist inevitably some uncertainties caused by the existence of modeling errors, external disturbance, and parameter fluctuation, which would lead to complex dynamic behaviors. Thus, it is important to investigate the robustness of neural networks against such uncertainties and deviations (see [18] and references therein). In [4-6], employing homeomorphism techniques, Lyapunov method, $H$-matrix and $M$ matrix theory, and linear matrix inequality (LMI) approach, Shao et al. established some sufficient conditions for the existence, uniqueness, and global robust exponential stability of the equilibrium point for the following interval Hopfield neural networks:

$$
\begin{array}{r}
\dot{u}_{i}(t)=-d_{i} u_{i}(t)+\sum_{j=1}^{n} a_{i j} f_{j}\left(u_{j}(t)\right) \\
+\sum_{j=1}^{n} b_{i j} f_{j}\left(u_{j}\left(t-\tau_{j}(t)\right)\right)-J_{i}, \\
i=1,2, \ldots, n,
\end{array}
$$

where $\tau_{j}(t)$ is time-varying delay which is variable with time due to the finite switching speed of amplifiers. Recently, the stability of neural networks with time-varying delays has been extensively investigated, and various sufficient conditions have been established for the global asymptotic and exponential stability in [9-13]. Generally, neural networks usually have a spatial extent due to the presence of a multitude of parallel pathways with a variety of axon sizes and lengths. It is desired to model them by introducing continuously distributed delays over a certain duration of time such that the distant past has less influence compared to the recent 
behavior of the state (see [14-16]). However, the distributed delays were not taken into account in system (1).

As an important neural networks, Cohen-Grossberg neural networks (CGNNs) include Hopfield neural networks, cellular neural networks, and other neural networks. CGNNs have aroused a tremendous surge of investigation in these years. Whereas, for the interval CGNNs, fewer robust stability results have been reported in contrast to the results on Hopfield neural networks [17-19]. On the other hand, the research of neural networks involves not only the dynamic analysis of equilibrium point but also that of the periodic oscillatory solution, which is very important in learning theory due to the fact that learning usually requires repetition $[20,21]$. Some important results for periodic solutions of neural networks have been obtained in $[7,22-27]$ and references therein. Motivated by the works of [4-6] and the discussions above, the objective of this paper is to investigate the global robust exponential stability and periodic solutions of the following CGNNs with time-varying and distributed delays:

$$
\begin{aligned}
& \dot{u}_{i}(t)=- \widetilde{\alpha}_{i}\left(u_{i}(t)\right) \\
& \times {\left[\widetilde{\beta}_{i}\left(u_{i}(t)\right)-\sum_{j=1}^{n} a_{i j} f_{j}\left(u_{j}(t)\right)\right.} \\
& \quad-\sum_{j=1}^{n} b_{i j} f_{j}\left(u_{j}\left(t-\tau_{j}(t)\right)\right) \\
&\left.\quad-\sum_{j=1}^{n} c_{i j} \int_{-\infty}^{t} k_{j}(t-s) f_{j}\left(u_{j}(s)\right) \mathrm{d} s+J_{i}(t)\right], \\
& i=1,2, \ldots, n,
\end{aligned}
$$

or equivalently

$$
\begin{array}{r}
\dot{u}(t)=-\widetilde{\alpha}(u(t))[\widetilde{\beta}(u(t))-A f(u(t))-B f(u(t-\tau(t))) \\
\left.-C \int_{-\infty}^{t} K(t-s) f(u(s)) \mathrm{d} s+J(t)\right],
\end{array}
$$

where

$$
\begin{gathered}
u(t)=\left(u_{1}(t), \ldots, u_{n}(t)\right)^{T}, \\
\tilde{\alpha}(u(t))=\operatorname{diag}\left(\widetilde{\alpha}_{1}\left(u_{1}(t)\right), \ldots, \widetilde{\alpha}_{n}\left(u_{n}(t)\right)\right), \\
\widetilde{\beta}(u(t))=\left(\widetilde{\beta}_{1}\left(u_{1}(t)\right), \ldots, \widetilde{\beta}_{n}\left(u_{n}(t)\right)\right)^{T}, \\
f(u(t))=\left(f_{1}\left(u_{1}(t)\right), \ldots, f_{n}\left(u_{n}(t)\right)\right)^{T}, \\
f(u(t-\tau(t)))=\left(f_{1}\left(u_{1}\left(t-\tau_{1}(t)\right)\right), \ldots, f_{n}\left(u_{n}\left(t-\tau_{n}(t)\right)\right)\right)^{T}, \\
K(t)=\operatorname{diag}\left(k_{1}(t), \ldots, k_{n}(t)\right), \\
A=\left(a_{i j}\right)_{n \times n^{\prime}} \quad B=\left(b_{i j}\right)_{n \times n}, \quad C=\left(c_{i j}\right)_{n \times n}, \\
J(t)=\left(J_{1}(t), \ldots, J_{n}(t)\right)^{T},
\end{gathered}
$$

where $u_{i}(t)$ denotes the state of the $i$ th neuron at time $t, \widetilde{\alpha}_{i}\left(u_{i}(t)\right)$ denotes a positive, continuous, and bounded amplification function; that is, $0<\underline{\alpha}_{i} \leq \widetilde{\alpha}_{i}\left(u_{i}(t)\right) \leq \bar{\alpha}_{i}<+\infty$, $\widetilde{\beta}_{i}\left(u_{i}(t)\right)$ denotes an appropriate behaved function, $f_{j}\left(u_{j}(t)\right)$ denotes the activation function, $\tau_{j}(t)$ denotes the timevarying delay associated with the $j$ th neuron, satisfying $0 \leq$ $\tau_{j}(t) \leq \tau$ and $0 \leq \dot{\tau}_{j}(t) \leq \delta<1, k_{j}(t)>0$ represents the delay kernel function, which is a real-valued continuous function, $A$ is the connection weight matrix, $B$ is the timevarying delayed connection weight matrix, $C$ is the infinite distributed delayed connection weight matrix, $J_{i}(t)$ is the external input bias. The coefficients $a_{i j}, b_{i j}$, and $c_{i j}$ can be intervalised as follows:

$$
\begin{aligned}
A_{I} & =[\underline{A}, \bar{A}] \\
& =\left\{A=\left(a_{i j}\right)_{n \times n}: \underline{a}_{i j} \leq a_{i j} \leq \bar{a}_{i j}, i, j=1,2, \ldots, n\right\}, \\
B_{I} & =[\underline{B}, \bar{B}] \\
& =\left\{B=\left(b_{i j}\right)_{n \times n}: \underline{b}_{i j} \leq b_{i j} \leq \bar{b}_{i j}, i, j=1,2, \ldots, n\right\}, \\
C_{I} & =[\underline{C}, \bar{C}] \\
& =\left\{C=\left(c_{i j}\right)_{n \times n}: \underline{c}_{i j} \leq c_{i j} \leq \bar{c}_{i j}, i, j=1,2, \ldots, n\right\},
\end{aligned}
$$

where for $X=A, B, C, \underline{X}=\left(\underline{x}_{i j}\right)_{n \times n}, \bar{X}=\left(\bar{x}_{i j}\right)_{n \times n}$. Denote $B^{*}=(\bar{B}+\underline{B}) / 2$ and $B_{*}=(\bar{B}-\underline{B}) / 2$. Clearly, $B_{*}$ is a nonnegative matrix and the interval matrix $[\underline{B}, \bar{B}]=\left[B^{*}-B_{*}, B^{*}+B_{*}\right]$. Consequently, $B=B^{*}+\Delta B, \Delta B \in\left[-B_{*}, B_{*}\right]$. $C^{*}$ and $C_{*}$ are defined correspondingly.

Throughout this paper, we make the following assumptions.

(H1) For the behaved functions $\widetilde{\beta}_{i}(\cdot)(i=1,2, \ldots, n)$, there exist constants $\gamma_{i}>0$ such that

$$
\frac{\widetilde{\beta}_{i}(x)-\tilde{\beta}_{i}(y)}{x-y} \geq \gamma_{i}>0, \quad \forall x, y \in \mathbb{R}, x \neq y .
$$

(H2) For the activation functions $f_{i}(\cdot)(i=1,2, \ldots, n)$, there exist constants $l_{i}>0$ such that

$$
0 \leq \frac{f_{i}(x)-f_{i}(y)}{x-y} \leq l_{i}, \quad \forall x, y \in \mathbb{R}, \quad x \neq y .
$$

(H3) The delay kernels $k_{j}(\cdot)(j=1,2, \ldots, n)$ satisfy

$$
\int_{0}^{\infty} k_{j}(s) \mathrm{d} s=1, \quad \int_{0}^{\infty} k_{j}(s) e^{\mu s} \mathrm{~d} s<\infty,
$$

for some positive constant $\mu$.

A typical example of such delay kernels is given by $k_{j}(s)=$ $s^{r} / r ! \gamma_{j}^{r+1} e^{-\gamma_{j} s}$ for $s \in[0, \infty)$, where $\gamma_{j} \in[0, \infty), r \in\{0,1, \ldots$, $n\}$, which are called the Gamma Memory Filter in [28].

The organization of this paper is as follows. In Section 2, some preliminaries are given. In Section 3, sufficient conditions are presented for the existence, uniqueness, and global robust exponential stability of the equilibrium point for system (2) with the external constant input bias (i.e., $J_{i}(t) \equiv$ $J_{i}, J_{i}$ is a constant). In Section 4, sufficient conditions are given which guarantee the uniqueness and global exponential 
stability of periodic solutions for system (2) when the timevarying delay $\tau_{i}(t)$ and the external input bias $J_{i}(t)$ are continuously periodic functions. Numerical examples are provided to illustrate the effectiveness of the obtained results in Section 5. A concluding remark is given in Section 6 to end this work.

\section{Preliminaries}

We give some preliminaries in this section. Denote $\Gamma=\operatorname{diag}\left(\gamma_{1}, \gamma_{2}, \ldots, \gamma_{n}\right), L=\operatorname{diag}\left(l_{1}, l_{2}, \ldots, l_{n}\right), \underline{\alpha}=\operatorname{diag}\left(\underline{\alpha}_{1}\right.$, $\left.\underline{\alpha}_{2}, \ldots, \underline{\alpha}_{n}\right)$, and $\widehat{x}_{i j}=\max \left\{\left|\underline{x}_{i j}\right|,\left|\bar{x}_{i j}\right|\right\}$. For a vector $x=\left(x_{1}\right.$, $\left.x_{2}, \ldots, x_{n}\right),\|x\|_{r}=\left(\sum_{i=1}^{n}\left|x_{i}\right|^{r}\right)^{1 / r}$ and for any $\varphi(s)=\left(\varphi_{1}(s)\right.$, $\left.\ldots, \varphi_{n}(s)\right), \quad s \quad \in \quad(-\infty, 0],\|\varphi(s)\|_{r}=$ $\sup _{s \in(-\infty, 0]}\left(\sum_{i=1}^{n}\left|\varphi_{i}(s)\right|^{r}\right)^{1 / r}$. For a matrix $A=\left(a_{i j}\right)_{n \times n}$, $A^{T}$ denotes the transpose; $A^{-1}$ denotes the inverse; $A>(\geq) 0$ means that $A$ is a symmetric positive definite (semidefinite) matrix; $\lambda_{\max }(A)$ and $\lambda_{\min }(A)$ denote the largest and the smallest eigenvalues of $A$, respectively; and $\|A\|_{2}=\sqrt{\lambda_{\max }\left(A^{T} A\right)}$ denotes the spectral norm of $A . I$ denotes the identity matrix. * denotes the symmetric block in a symmetric matrix.

Definition 1 (see [29]). The neural network (2) with the parameter ranges defined by (5) is globally robustly exponentially stable, if for each $A \in A_{I}, B \in B_{I}, C \in C_{I}$, and $J$, system (2) has a unique equilibrium point $u^{*}=\left(u_{1}^{*}, u_{2}^{*}, \ldots, u_{n}^{*}\right)^{T}$, and there exist constants $a \geq 1$ and $\varepsilon>0$ such that

$$
\left\|u(t)-u^{*}\right\| \leq a\left\|\phi(\theta)-u^{*}\right\| e^{-\varepsilon t}, \quad \forall t>0,
$$

where $u(t)=\left(u_{1}(t), u_{2}(t), \ldots, u_{n}(t)\right)^{T}$ is a solution of system (2) with the initial value $u_{i}(\theta)=\phi_{i}(\theta), i=1,2, \ldots, n, \theta \in$ $(-\infty, 0]$, and $\phi(\theta)=\left(\phi_{1}(\theta), \phi_{2}(\theta), \ldots, \phi_{n}(\theta)\right)$.

Definition 2 (see [30]). Let $Z_{n}=\left\{A=\left(a_{i j}\right)_{n \times n} \in M_{n}(\mathbb{R})\right.$ : $a_{i j} \leq 0$ if $\left.i \neq j, i, j=1,2, \ldots, n\right\}$, where $M_{n}(\mathbb{R})$ denotes the set of all $n \times n$ matrices with entries from $\mathbb{R}$. Then a matrix $A$ is called an $M$-matrix if $A \in Z_{n}$ and all successive principal minors of $A$ are positive.

Definition 3 (see [30]). An $n \times n$ matrix $A=\left(a_{i j}\right)_{n \times n}$ is said to be an $H$-matrix if its comparison matrix $M(A)=\left(m_{i j}\right)_{n \times n}$ is an $M$-matrix, where $m_{i j}= \begin{cases}\left|a_{i i}\right|, & \text { if } i=j, \\ -\left|a_{i j}\right|, & \text { if } i \neq j .\end{cases}$

Lemma 4 (see [31]). For any vectors $x, y \in \mathbb{R}^{n}$ and positive definite matrix $G \in \mathbb{R}^{n \times n}$, the following inequality holds: $2 x^{T} y \leq x^{T} G x+y^{T} G^{-1} y$.

Lemma 5 (see [30]). Let $A, B \in Z_{n}$. If $A$ is an $M$-matrix and the elements of matrices $A$ and $B$ satisfy the inequalities $a_{i j} \leq$ $b_{i j}, i, j=1,2, \ldots, n$, then $B$ is an M-matrix.

Lemma 6 (see [30]). The following LMI: $\left(\begin{array}{cc}Q(x) & S(x) \\ S^{T}(x) & R(x)\end{array}\right)>0$, where $Q(x)=Q^{T}(x), R(x)=R^{T}(x)$, is equivalent to $R(x)>$ 0 and $Q(x)-S(x) R^{-1}(x) S^{T}(x)>0$ or $Q(x)>0$ and $R(x)-$ $S^{T}(x) Q^{-1}(x) S(x)>0$.
Lemma 7 (see [32]). Suppose that the neural network parameters are defined by (5), and

$$
\Xi=\left(\begin{array}{ccc}
\Phi-S & -P B_{*} & -P C_{*} \\
* & (1-\delta) Q & 0 \\
* & * & R
\end{array}\right)>0,
$$

where $P=\operatorname{diag}\left(p_{1}, p_{2}, \ldots, p_{n}\right), Q=\operatorname{diag}\left(q_{1}, q_{2}, \ldots, q_{n}\right)$, and $R=\operatorname{diag}\left(r_{1}, r_{2}, \ldots, r_{n}\right)$ are positive diagonal matrices, $\Phi=$ $\left(\Phi_{i j}\right)_{n \times n}=2 P \Gamma L^{-1}-((2-\delta) /(1-\delta))\left\|P B^{*}\right\|_{2} I-2\left\|P C^{*}\right\|_{2} I-Q-R$, $S=\left(s_{i j}\right)_{n \times n}$ with

$$
s_{i j}= \begin{cases}2 p_{i} \bar{a}_{i i}, & \text { if } i=j, \\ \max \left\{\left|p_{i} \underline{a}_{i j}+p_{j} \underline{a}_{j i}\right|,\left|p_{i} \bar{a}_{i j}+p_{j} \bar{a}_{j i}\right|\right\}, & \text { if } i \neq j\end{cases}
$$

Then, for all $A \in A_{I}, B \in B_{I}$, and $C \in C_{I}$, we have

$$
\Theta=\left(\begin{array}{ccc}
\Phi-S^{\prime} & -P \Delta B & -P \Delta C \\
* & (1-\delta) Q & 0 \\
* & * & R
\end{array}\right)>0
$$

where $S^{\prime}=\left(s_{i j}^{\prime}\right)_{n \times n}=P A+A^{T} P$.

\section{Global Robust Exponential Stability of the Equilibrium Point}

In this section, in system (2), we assume that the external input bias $J_{i}(t) \equiv J_{i}, J_{i}$ is a constant $(i=1,2, \ldots, n)$, and we will give a new sufficient condition for the existence and uniqueness of the equilibrium point for system (2) and analyze the global robust exponential stability of the equilibrium point.

Theorem 8. Under assumptions (H1) and (H2), if there exist positive diagonal matrices $P=\operatorname{diag}\left(p_{1}, p_{2}, \ldots, p_{n}\right), Q=$ $\operatorname{diag}\left(q_{1}, q_{2}, \ldots, q_{n}\right)$, and $R=\operatorname{diag}\left(r_{1}, r_{2}, \ldots, r_{n}\right)$ such that $\Xi>$ 0 , where $\Xi$ is defined by (10), then system (2) has a unique equilibrium point.

The proof of Theorem 8 is similar to that in [32], therefore we omit it here.

Let $u^{*}=\left(u_{1}^{*}, u_{2}^{*}, \ldots, u_{n}^{*}\right)^{T}$ be the equilibrium point of system (2). By coordinate transformation $v(t)=u(t)-u^{*}$, one can transform system (2) into the following system

$$
\begin{aligned}
\dot{v}_{i}(t)= & -\alpha_{i}\left(v_{i}(t)\right) \\
& \times\left[\beta_{i}\left(v_{i}(t)\right)-\sum_{j=1}^{n} a_{i j} g_{j}\left(v_{j}(t)\right)\right. \\
& -\sum_{j=1}^{n} b_{i j} g_{j}\left(v_{j}\left(t-\tau_{j}(t)\right)\right) \\
& \left.-\sum_{j=1}^{n} c_{i j} \int_{-\infty}^{t} k_{j}(t-s) g_{j}\left(v_{j}(s)\right) \mathrm{d} s\right], \\
& i=1,2, \cdots, n,
\end{aligned}
$$


or equivalently

$$
\begin{aligned}
\dot{v}(t)= & -\alpha(v(t)) \\
& \times[\beta(v(t))-A g(v(t))-B g(v(t-\tau(t))) \\
& \left.-C \int_{-\infty}^{t} K(t-s) g(v(s)) \mathrm{d} s\right],
\end{aligned}
$$

where $v(t)=\left(v_{1}(t), \ldots, v_{n}(t)\right)^{T}, \alpha(v(t))=\operatorname{diag}\left(\alpha_{1}\left(v_{1}(t)\right), \ldots\right.$, $\left.\alpha_{n}\left(v_{n}(t)\right)\right)$ with $\alpha_{i}\left(v_{i}(t)\right)=\tilde{\alpha}_{i}\left(v_{i}(t)+u_{i}^{*}\right), \beta(v(t))=$ $\left(\beta_{1}\left(v_{1}(t)\right), \ldots, \beta_{n}\left(v_{n}(t)\right)\right)^{T}$ with $\beta_{i}\left(v_{i}(t)\right)=\widetilde{\beta}_{i}\left(v_{i}(t)+u_{i}^{*}\right)-$ $\widetilde{\beta}_{i}\left(u_{i}^{*}\right), g(v(t))=\left(g_{1}\left(v_{1}(t)\right), \ldots, g_{n}\left(v_{n}(t)\right)\right)^{T}, g(v(t-\tau(t)))=$ $\left(g_{1}\left(v_{1}\left(t-\tau_{1}(t)\right)\right), \ldots, g_{n}\left(v_{n}\left(t-\tau_{n}(t)\right)\right)\right)^{T}$ with $g_{j}\left(v_{j}(t)\right)=$ $f_{j}\left(v_{j}(t)+u_{j}^{*}\right)-f_{j}\left(u_{j}^{*}\right)$.

Theorem 9. Under assumptions (H1)-(H3), if there exist positive diagonal matrices $P=\operatorname{diag}\left(p_{1}, p_{2}, \ldots, p_{n}\right), Q=$ $\operatorname{diag}\left(q_{1}, q_{2}, \ldots, q_{n}\right)$, and $R=\operatorname{diag}\left(r_{1}, r_{2}, \ldots, r_{n}\right)$ such that $\Xi>$ 0 , where $\Xi$ is defined by (10), then the equilibrium point of system (2) is globally robustly exponentially stable.

Proof. Define a Lyapunov functional: $V(t)=\sum_{i=1}^{4} V_{i}(t)$, where

$$
\begin{gathered}
V_{1}(t)=2 h e^{\varepsilon t} \sum_{i=1}^{n} \int_{0}^{v_{i}(t)} \frac{s}{\alpha_{i}(s)} \mathrm{d} s, \\
V_{2}(t)=2 e^{\varepsilon t} \sum_{i=1}^{n} p_{i} \int_{0}^{v_{i}(t)} \frac{g_{i}(s)}{\alpha_{i}(s)} \mathrm{d} s \\
V_{3}(t)=\sum_{i=1}^{n}\left(q_{i}+\left\|P B^{*}\right\|_{2}\right) \int_{t-\tau_{i}(t)}^{t} e^{\varepsilon(\tau+s)} g_{i}^{2}\left(v_{i}(s)\right) \mathrm{d} s, \\
V_{4}(t)=\sum_{i=1}^{n}\left(r_{i}+\left\|P C^{*}\right\|_{2}\right) \int_{0}^{+\infty} k_{i}(\xi) e^{\varepsilon \xi} \int_{t-\xi}^{t} e^{\varepsilon s} g_{i}^{2}\left(v_{i}(s)\right) \mathrm{d} s \mathrm{~d} \xi .
\end{gathered}
$$

Calculating the derivative of $V(t)$ along the trajectories of system (13), we obtain that

$$
\begin{aligned}
& \dot{V}_{1}(t)=2 h \varepsilon e^{\varepsilon t} \sum_{i=1}^{n} \int_{0}^{v_{i}(t)} \frac{s}{\alpha_{i}(s)} \mathrm{d} s-2 h e^{\varepsilon t} \\
& \times \sum_{i=1}^{n} v_{i}(t)\left[\beta_{i}\left(v_{i}(t)\right)-\sum_{j=1}^{n} a_{i j} g_{j}\left(v_{j}(t)\right)\right. \\
& \quad-\sum_{j=1}^{n} b_{i j} g_{j}\left(v_{j}\left(t-\tau_{j}(t)\right)\right) \\
&\left.\quad-\sum_{j=1}^{n} c_{i j} \int_{-\infty}^{t} k_{j}(t-s) g_{j}\left(v_{j}(s)\right) \mathrm{d} s\right] \\
& \leq h \varepsilon e^{\varepsilon t} \sum_{i=1}^{n} \frac{1}{\alpha} \underline{\alpha}_{i}^{2}(t)-2 h e^{\varepsilon t} \sum_{i=1}^{n} \gamma_{i} v_{i}^{2}(t)+2 h e^{\varepsilon t}
\end{aligned}
$$

$$
\begin{aligned}
& \times \sum_{i=1}^{n} v_{i}(t)\left[\sum_{j=1}^{n} a_{i j} g_{j}\left(v_{j}(t)\right)\right. \\
& +\sum_{j=1}^{n} b_{i j} g_{j}\left(v_{j}\left(t-\tau_{j}(t)\right)\right) \\
& \left.+\sum_{j=1}^{n} c_{i j} \int_{-\infty}^{t} k_{j}(t-s) g_{j}\left(v_{j}(s)\right) \mathrm{d} s\right] \\
& =h \varepsilon e^{\varepsilon t} v^{T}(t) \underline{\alpha}^{-1} v(t)+2 h e^{\varepsilon t} v^{T}(t) \\
& \times[-\Gamma v(t)+A g(v(t))+B g(v(t-\tau(t))) \\
& \left.+C \int_{-\infty}^{t} K(t-s) g(v(s)) \mathrm{d} s\right], \\
& \dot{V}_{2}(t)=2 \varepsilon e^{\varepsilon t} \sum_{i=1}^{n} p_{i} \int_{0}^{v_{i}(t)} \frac{g_{i}(s)}{\alpha_{i}(s)} d s-2 e^{\varepsilon t} \\
& \times \sum_{i=1}^{n} p_{i} g_{i}\left(v_{i}(t)\right) \\
& \times\left[\beta_{i}\left(v_{i}(t)\right)-\sum_{j=1}^{n} a_{i j} g_{j}\left(v_{j}(t)\right)\right. \\
& -\sum_{j=1}^{n} b_{i j} g_{j}\left(v_{j}\left(t-\tau_{j}(t)\right)\right) \\
& \left.-\sum_{j=1}^{n} c_{i j} \int_{-\infty}^{t} k_{j}(t-s) g_{j}\left(v_{j}(s)\right) \mathrm{d} s\right] \\
& \leq \varepsilon e^{\varepsilon t} \sum_{i=1}^{n} \frac{p_{i} l_{i}}{\underline{\alpha}_{i}} v_{i}^{2}(t)-2 e^{\varepsilon t} \sum_{i=1}^{n} p_{i} \gamma_{i} g_{i}\left(v_{i}(t)\right) v_{i}(t)+2 e^{\varepsilon t} \\
& \times \sum_{i=1}^{n} p_{i} g_{i}\left(v_{i}(t)\right) \\
& \times\left[\sum_{j=1}^{n} a_{i j} g_{j}\left(v_{j}(t)\right)+\sum_{j=1}^{n} b_{i j} g_{j}\left(v_{j}\left(t-\tau_{j}(t)\right)\right)\right. \\
& \left.+\sum_{j=1}^{n} c_{i j} \int_{-\infty}^{t} k_{j}(t-s) g_{j}\left(v_{j}(s)\right) \mathrm{d} s\right] \\
& =\varepsilon e^{\varepsilon t} v^{T}(t) P L \underline{\alpha}^{-1} v(t)-2 e^{\varepsilon t} g^{T}(v(t)) P \Gamma v(t) \\
& +2 e^{\varepsilon t} g^{T}(v(t)) \\
& \times[P A g(v(t))+\operatorname{PBg}(v(t-\tau(t))) \\
& \left.+P C \int_{-\infty}^{t} K(t-s) g(v(s)) \mathrm{d} s\right] \\
& \leq \varepsilon e^{\varepsilon t} v^{T}(t) P L \underline{\alpha}^{-1} v(t)-2 e^{\varepsilon t} g^{T}(v(t)) P \Gamma L^{-1} g(v(t)) \\
& +2 e^{\varepsilon t} g^{T}(v(t)) P A g(v(t))+e^{\varepsilon t}\left\|P B^{*}\right\|_{2} \\
& \times\left(\frac{1}{1-\delta}\|g(v(t))\|_{2}^{2}+(1-\delta)\|g(v(t-\tau(t)))\|_{2}^{2}\right) \\
& +e^{\varepsilon t}\left\|P C^{*}\right\|_{2}
\end{aligned}
$$




$$
\begin{aligned}
& \times\left(\|g(v(t))\|_{2}^{2}+\left\|\int_{-\infty}^{t} K(t-s) g(v(s)) \mathrm{d} s\right\|_{2}^{2}\right) \\
& +2 e^{\varepsilon t} g^{T}(v(t)) P \Delta B g(v(t-\tau(t))) \\
& +2 e^{\varepsilon t} g^{T}(v(t)) P \Delta C \int_{-\infty}^{t} K(t-s) g(v(s)) \mathrm{d} s, \\
\dot{V}_{3}(t)= & \sum_{i=1}^{n}\left(q_{i}+\left\|P B^{*}\right\|_{2}\right) \\
& \times\left[e^{\varepsilon(t+\tau)} g_{i}^{2}\left(v_{i}(t)\right)-\left(1-\dot{\tau}_{i}(t)\right) e^{\varepsilon\left(t+\tau-\tau_{i}(t)\right)}\right. \\
\leq & e^{\varepsilon(t+\tau)} g^{T}(v(t)) Q g(v(t))+e^{\varepsilon(t+\tau)}\left\|P B^{*}\right\|_{2}\|g(v(t))\|_{2}^{2} \\
& -(1-\delta) e^{\varepsilon t} g^{T}(v(t-\tau(t))) Q g(v(t-\tau(t))) \\
& -(1-\delta) e^{\varepsilon t}\left\|P B^{*}\right\|_{2}\|g(v(t-\tau(t)))\|_{2}^{2}, \\
\dot{V}_{4}(t)= & \sum_{i=1}^{n}\left(r_{i}+\left\|P C^{*}\right\|_{2}\right) \\
& \times \int_{0}^{+\infty} k_{i}(\xi) e^{\varepsilon(t+\xi)}\left[g_{i}^{2}\left(v_{i}(t)\right)-e^{-\varepsilon \xi} g_{i}^{2}\left(v_{i}(t-\xi)\right)\right] \mathrm{d} \xi \\
= & e^{\varepsilon t} \int_{0}^{+\infty} k_{i}(\xi) e^{\varepsilon \xi} \mathrm{d} \xi g^{T}(v(t))\left(R+\left\|P C^{*}\right\|_{2} I\right) g(v(t)) \\
& -e^{\varepsilon t} \sum_{i=1}^{n}\left(r_{i}+\left\|P C^{*}\right\|_{2}\right) \int_{-\infty}^{t} k_{i}(t-s) g_{i}^{2}\left(v_{i}(s)\right) \mathrm{d} s \\
\leq & e^{\varepsilon t} \int_{0}^{+\infty} k_{i}(\xi) e^{\varepsilon \xi} \mathrm{d} \xi g^{T}(v(t))\left(R+\left\|P C^{*}\right\|_{2} I\right) g(v(t)) \\
& -e^{\varepsilon t}\left(\int_{-\infty}^{t} K(t-s) g(v(s)) \mathrm{d} s\right)^{T}\left(R+\left\|P C^{*}\right\|_{2} I\right) \\
& \times\left(\int_{-\infty}^{t} K(t-s) g(v(s)) \mathrm{d} s\right) . \\
&
\end{aligned}
$$

Therefore, one can deduce that

$$
\begin{aligned}
\dot{V}(t) \leq & \varepsilon e^{\varepsilon t} v^{T}(t)\left(h \underline{\alpha}^{-1}+P L \underline{\alpha}^{-1}\right) v(t) \\
& +e^{\varepsilon(t+\tau)} g^{T}(v(t))\left(Q+\left\|P B^{*}\right\|_{2} I\right) g(v(t))+e^{\varepsilon t} \\
& \times \int_{0}^{+\infty} k_{i}(s) e^{\varepsilon s} \mathrm{~d} s g^{T}(v(t))\left(R+\left\|P C^{*}\right\|_{2} I\right) g(v(t)) \\
& \quad-2 h e^{\varepsilon t} v^{T}(t) \Gamma v(t) \\
& -e^{\varepsilon t} g^{T}(v(t)) \\
\times & \left(2 P \Gamma L^{-1}-2 P A-\frac{1}{1-\delta}\left\|P B^{*}\right\|_{2}-\left\|P C^{*}\right\|_{2}\right) \\
\times & g(v(t))-e^{\varepsilon t}(1-\delta) g^{T}(v(t-\tau(t))) Q g(v(t-\tau(t))) \\
- & e^{\varepsilon t}\left(\int_{-\infty}^{t} K(t-s) g(v(s)) \mathrm{d} s\right)^{T} \\
\times & R\left(\int_{-\infty}^{t} K(t-s) g(v(s)) \mathrm{d} s\right)+2 h e^{\varepsilon t} v^{T}(t) \\
\times & (A g(v(t))+B g(v(t-\tau(t))) \\
& \left.\quad+C \int_{-\infty}^{t} K(t-s) g(v(s)) \mathrm{d} s\right)
\end{aligned}
$$

$$
\begin{aligned}
& +2 e^{\varepsilon t} g^{T}(v(t)) \\
& \times(P \Delta B g(v(t-\tau(t))) \\
& \left.\quad+P \Delta C \int_{-\infty}^{t} K(t-s) g(v(s)) \mathrm{d} s\right) \\
& =\varepsilon e^{\varepsilon t} v^{T}(t)\left(h \underline{\alpha}^{-1}+P L \underline{\alpha}^{-1}\right) v(t) \\
& +e^{\varepsilon t}\left(e^{\varepsilon \tau}-1\right) g^{T}(v(t))\left(Q+\left\|P B^{*}\right\|_{2} I\right) g(v(t)) \\
& +e^{\varepsilon t}\left(\int_{0}^{+\infty} k_{i}(s) e^{\varepsilon s} d s-1\right) g^{T}(v(t))\left(R+\left\|P C^{*}\right\|_{2} I\right) \\
& \times g(v(t))-e^{\varepsilon t} w^{T}(t) \Psi w(t),
\end{aligned}
$$

where

$$
\begin{aligned}
\Psi= & \left(\begin{array}{cccc}
2 h \Gamma & -h A & -h B & -h C \\
* & \Phi-S^{\prime} & -P \Delta B & -P \Delta C \\
* & * & (1-\delta) Q & 0 \\
* & * & * & R
\end{array}\right), \\
w(t)= & \left(v^{T}(t) g^{T}(v(t)) g^{T}(v(t-\tau(t)))\right. \\
& \left.\times\left(\int_{-\infty}^{t} K(t-s) g(v(s)) \mathrm{d} s\right)^{T}\right)^{T} .
\end{aligned}
$$

Denote $\Upsilon=\left(\begin{array}{lll}A & B & C\end{array}\right)$. From Lemma $6, \Psi>0$ is equivalent to $\Theta-(h / 2) \Upsilon^{T} \Gamma^{-1} \Upsilon>0$, where $\Theta$ is defined in (12). By Lemma 7, we have $\Theta>0$. Letting $0<h<$ $2 \min _{1 \leq i \leq n}\left\{\gamma_{i}\right\} \lambda_{\min }(\Theta) / \lambda_{\max }\left(\Upsilon^{T} \Upsilon\right)$, we can derive that

$$
\Theta-\frac{h}{2} \Upsilon^{T} \Gamma^{-1} \Upsilon \geq \Theta-\frac{h}{2 \min _{1 \leq i \leq n}\left\{\gamma_{i}\right\}} \Upsilon^{T} \Upsilon>0,
$$

which yields $\Psi>0$.

From assumption (H3), we can choose a constant $\varepsilon_{3}$ sufficiently small satisfying $0<\varepsilon_{3}<\mu$ and

$$
\int_{0}^{\infty} k_{i}(s) e^{\varepsilon_{3} s} \mathrm{~d} s \leq \frac{\lambda_{\min }(\Psi)}{2 \max _{1 \leq i \leq n}\left\{r_{i}+\left\|P C^{*}\right\|_{2}\right\}}+1, \quad 1 \leq i \leq n .
$$

Choosing $0<\varepsilon<\min _{1 \leq i \leq 3}\left\{\varepsilon_{i}\right\}$ with

$$
\begin{gathered}
\varepsilon_{1}=\frac{\lambda_{\min }(\Psi)}{\max _{1 \leq i \leq n}\left\{\underline{\alpha}_{i}^{-1}\left(h+p_{i} l_{i}\right)\right\}}, \\
\varepsilon_{2}=\frac{1}{\tau} \ln \left(\frac{\lambda_{\min }(\Psi)}{2 \max _{1 \leq i \leq n}\left\{q_{i}+\left\|P B^{*}\right\|_{2}\right\}}+1\right),
\end{gathered}
$$

we get

$$
\begin{aligned}
\dot{V}(t) \leq & \varepsilon_{1} e^{\varepsilon t} v^{T}(t)\left(h \underline{\alpha}^{-1}+P L \underline{\alpha}^{-1}\right) v(t) \\
& +e^{\varepsilon t}\left(e^{\varepsilon_{2} \tau}-1\right) g^{T}(v(t))\left(Q+\left\|P B^{*}\right\|_{2} I\right) g(v(t)) \\
& +e^{\varepsilon t}\left(\int_{0}^{+\infty} k_{i}(s) e^{\varepsilon_{3} s} \mathrm{~d} s-1\right) g^{T}(v(t)) \\
& \times\left(R+\left\|P C^{*}\right\|_{2} I\right) g(v(t))-e^{\varepsilon t} w^{T}(t) \Psi w(t) \\
< & e^{\varepsilon t} \lambda_{\min }(\Psi)\left[v^{T}(t) v(t)+g^{T}(v(t)) g(v(t))\right] \\
& -e^{\varepsilon t} w^{T}(t) \Psi w(t) \leq 0 .
\end{aligned}
$$

Consequently, $V(t) \leq V(0)$ for all $t \geq 0$. 
On the other hand,

$$
\begin{aligned}
V(0)= & 2 h e^{\varepsilon t} \sum_{i=1}^{n} \int_{0}^{v_{i}(0)} \frac{s}{\alpha_{i}(s)} d s+2 \sum_{i=1}^{n} p_{i} \int_{0}^{v_{i}(0)} \frac{g_{i}(s)}{\alpha_{i}(s)} \mathrm{d} s \\
& +\sum_{i=1}^{n}\left(q_{i}+\left\|P B^{*}\right\|_{2}\right) \\
& \times \int_{-\tau_{i}(0)}^{0} e^{\varepsilon(\tau+s)} g_{i}^{2}\left(v_{i}(s)\right) \mathrm{d} s+\sum_{i=1}^{n}\left(r_{i}+\left\|P C^{*}\right\|_{2}\right) \\
& \times \int_{0}^{+\infty} k_{i}(\xi) e^{\varepsilon \xi} \int_{-\xi}^{0} e^{\varepsilon s} g_{i}^{2}\left(v_{i}(s)\right) \mathrm{d} s \mathrm{~d} \xi \\
\leq & \max _{1 \leq i \leq n}\left\{\frac{h_{i}}{\underline{\alpha}_{i}}\right\}\|v(0)\|_{2}^{2}+\max _{1 \leq i \leq n}\left\{\frac{p_{i} l_{i}}{\alpha_{i}}\right\}\|v(0)\|_{2}^{2} \\
& +\tau e^{\varepsilon \tau} \max _{1 \leq i \leq n}\left\{l_{i}^{2}\left(q_{i}+\left\|P B^{*}\right\|_{2}\right)\right\} \times \sup _{-\tau \leq \theta \leq 0}\|v(\theta)\|_{2}^{2} \\
& +\frac{1}{2 \varepsilon} \max _{1 \leq i \leq n}\left\{l_{i}^{2}\right\} \lambda_{\min }(\Psi) \quad \sup _{-\infty<\theta \leq 0}\|v(\theta)\|_{2}^{2} \\
\leq & a \sup _{-\infty<\theta \leq 0}\|v(\theta)\|_{2}^{2}, \quad
\end{aligned}
$$

where $a=\max _{1 \leq i \leq n}\left\{h_{i} / \underline{\alpha}_{i}\right\}+\max _{1 \leq i \leq n}\left\{p_{i} l_{i} / \underline{\alpha}_{i}\right\}+\tau e^{\varepsilon \tau}$ $\max _{1 \leq i \leq n}\left\{l_{i}^{2}\left(q_{i}+\left\|P B^{*}\right\|_{2}\right)\right\}+(1 / 2 \varepsilon) \max _{1 \leq i \leq n}\left\{l_{i}^{2}\right\} \lambda_{\min }(\Psi)$. Hence, $\left(h / \max _{1 \leq i \leq n}\left\{\bar{\alpha}_{i}\right\}\right) e^{\varepsilon t}\|v(t)\|_{2}^{2} \leq V(t) \leq V(0) \leq$ $\operatorname{asup}_{-\infty<\theta \leq 0}\|v(\theta)\|_{2}^{2}$; that is,

$$
\left\|u(t)-u^{*}\right\|_{2} \leq \sqrt{\frac{a \max _{1 \leq i \leq n}\left\{\bar{\alpha}_{i}\right\}}{h}}\left\|\phi(\theta)-u^{*}\right\|_{2} e^{-\varepsilon t / 2}, \quad t>0 .
$$

Combining Theorem 8, we get that system (2) is globally robustly exponentially stable. The proof is complete.

Remark 10. Letting $P=p I$ be a positive scalar matrix in Theorem 9 , we can get a robust exponential stability criterion based on LMI.

Remark 11. If $\widetilde{\alpha}_{i}\left(u_{i}(t)\right)=1, \widetilde{\beta}_{i}\left(u_{i}(t)\right)=d_{i} u_{i}(t), 0<\underline{d}_{i} \leq$ $d_{i} \leq \bar{d}_{i}$, and $c_{i j}=0$, system (2) turns into the interval Hopfield neural networks (1), which was studied in [4-6]. It can be seen that the main results in [4] is a special case of Theorem 9. Therefore, the obtained results in this paper improve the results in [4-6]. Also, our results generalize some previous ones in [33-35] as mentioned in [4]. In addition, in [19], the authors dealt with the robust exponential stability of CGNNs with time-varying delays. However, the distributed delays were not taken into account. Therefore, our results in this paper are more general than those reported in [19].

Remark 12. In previous works such as $[6,33-35],\left\|B_{*}\right\|_{2}$ is often used as a part to estimate the bounds for $\|B\|_{2}$. Considering that $B_{*}$ is a nonnegative matrix, we develop a new approach based on $\mathrm{H}$-matrix theory. The obtained robust stability criterion is in terms of the matrices $B_{*}$ and $B_{*}^{T}$, which can reduce the conservativeness of the robust results to some extent.

\section{Periodic Solutions of Interval CGNNs}

In this section, we consider the periodic solutions of system (2), in which $\tau_{i}(t)$ and $J_{i}(t)$ are continuously periodic functions with period $\omega$; that is, $\tau_{i}(t+\omega)=\tau_{i}(t), J_{i}(t+\omega)=$ $J_{i}(t)(i=1,2, \ldots, n)$.

Theorem 13. Under assumptions (H1)-(H3), system (2) has an $\omega$-periodic solution which is globally exponentially stable, if the following condition holds:

(H4) $\mathscr{M}=\Gamma-D$ is a nonsingular $M$-matrix, where

$$
D=\left(d_{i j}\right)_{n \times n}, \quad d_{i j}=l_{j}\left(\widehat{a}_{i j}+\frac{\widehat{b}_{i j}}{1-\delta}+\widehat{c}_{i j}\right) .
$$

Proof. Let $u_{i}(t, \phi)$ and $u_{i}(t, \psi)$ be two solutions of system (2) with initial values $\phi, \psi \in C\left((-\infty, 0], R^{n}\right)$, respectively. Since $\mathscr{M}$ is a nonsingular $M$-matrix, $\mathscr{M}^{T}$ is also a nonsingular $M$ matrix. It is well known that there exists a positive vector $p=$ $\left(\mu_{1}, \ldots, \mu_{n}\right)^{T}$ such that $\mathscr{M}^{T} p>0$; that is,

$$
\mu_{i} \gamma_{i}-\sum_{j=1}^{n} \mu_{j} l_{i}\left(\widehat{a}_{j i}+\frac{\widehat{b}_{j i}}{1-\delta}+\widehat{c}_{j i}\right)>0, \quad i=1,2, \ldots, n
$$

We can choose a constant $\varepsilon>0$ sufficiently small such that

$$
\begin{aligned}
F_{i}(\varepsilon)= & \mu_{i}\left(\gamma_{i}-\frac{\varepsilon}{\underline{\alpha}_{i}}\right) \\
& -\sum_{j=1}^{n} \mu_{j} l_{i}\left(\widehat{a}_{j i}+\frac{\widehat{b}_{j i}}{1-\delta} e^{\varepsilon \tau}+\widehat{c}_{j i} \int_{0}^{+\infty} k_{i}(\xi) e^{\varepsilon \xi} \mathrm{d} \xi\right) \\
> & 0, \quad i=1,2, \ldots, n .
\end{aligned}
$$

Denote $X_{i}(t)=\left|u_{i}(t, \phi)-u_{i}(t, \psi)\right|$. Define a Lyapunov functional

$$
W(t)=\sum_{i=1}^{n} \mu_{i}\left(W_{1 i}(t)+W_{2 i}(t)+W_{3 i}(t)\right)
$$

where

$$
\begin{gathered}
W_{1 i}(t)=e^{\varepsilon t} \operatorname{sgn}\left(u_{i}(t, \phi)-u_{i}(t, \psi)\right) \int_{u_{i}(t, \psi)}^{u_{i}(t, \phi)} \frac{1}{\widetilde{\alpha}_{i}(s)} \mathrm{d} s, \\
W_{2 i}(t)=\sum_{j=1}^{n} \frac{l_{j} \widehat{b}_{i j}}{1-\delta} \int_{t-\tau_{j}(t)}^{t} e^{\varepsilon(\tau+s)} X_{j}(s) \mathrm{d} s, \\
W_{3 i}(t)=\sum_{j=1}^{n} l_{j} \widehat{c}_{i j} \int_{0}^{+\infty} k_{j}(\xi) e^{\varepsilon \xi} \int_{t-\xi}^{t} e^{\varepsilon s} X_{j}(s) \mathrm{d} s \mathrm{~d} \xi .
\end{gathered}
$$


Calculating the upper right derivative of $W(t)$ along the solution of (2), together with assumptions $(\mathrm{H} 1)-(\mathrm{H} 3)$ and (28), we can derive that

$$
\begin{aligned}
& D^{+} W(t) \\
& \leq e^{\varepsilon t} \sum_{i=1}^{n} \mu_{i}\left[\left(\frac{\varepsilon}{\underline{\alpha}_{i}}-\gamma_{i}\right) X_{i}(t)\right. \\
& +\sum_{j=1}^{n} l_{j}\left(\widehat{a}_{i j}+\frac{\widehat{b}_{i j}}{1-\delta} e^{\varepsilon \tau}+\widehat{c}_{i j}\right. \\
& \left.\left.\times \int_{0}^{+\infty} k_{j}(\xi) e^{\varepsilon \xi} \mathrm{d} \xi\right) X_{j}(t)\right] \\
& =-e^{\varepsilon t} \sum_{i=1}^{n}\left[\mu_{i}\left(\gamma_{i}-\frac{\varepsilon}{\underline{\alpha}_{i}}\right)\right. \\
& -\sum_{j=1}^{n} \mu_{j} l_{i}\left(\widehat{a}_{j i}+\frac{\widehat{b}_{j i}}{1-\delta} e^{\varepsilon \tau}\right. \\
& \left.\left.+\widehat{c}_{j i} \int_{0}^{+\infty} k_{i}(\xi) e^{\varepsilon \xi} \mathrm{d} \xi\right)\right] X_{i}(t) \\
& =-e^{\varepsilon t} \sum_{i=1}^{n} F_{i}(\varepsilon) X_{i}(t) \leq 0 .
\end{aligned}
$$

Then, we have $W(t) \leq W(0)$ for $t \geq 0$. On the other hand, it can be readily seen that

$$
\begin{aligned}
& W(t) \geq m_{0} e^{\varepsilon t} \sum_{i=1}^{n}\left|u_{i}(t, \phi)-u_{i}(t, \psi)\right|, \\
& W(0) \leq M_{0} \sup _{s \in(-\infty, 0]} \sum_{i=1}^{n}\left|\phi_{i}(s)-\psi_{i}(s)\right|,
\end{aligned}
$$

in which

$$
\begin{gathered}
m_{0}=\min _{1 \leq i \leq n}\left\{\frac{\mu_{i}}{\bar{\alpha}_{i}}\right\}, \\
M_{0}=\max _{1 \leq i \leq n}\left\{\frac{\underline{\mu}_{i}}{\underline{\alpha}_{i}}+\sum_{j=1}^{n}\left[\frac{l_{j} \widehat{b}_{i j}\left(e^{\varepsilon \tau}-1\right)}{\varepsilon(1-\delta)}+\frac{l_{j} \widehat{c}_{i j}}{\varepsilon}\right.\right. \\
\left.\left.\times\left(\int_{0}^{+\infty} k_{j}(\xi) e^{\varepsilon \xi} \mathrm{d} \xi-1\right)\right]\right\} .
\end{gathered}
$$

Hence, $m_{0} e^{\varepsilon t} \sum_{i=1}^{n}\left|u_{i}(t, \phi)-u_{i}(t, \psi)\right| \leq W(t) \leq W(0) \leq$ $M_{0} \sup _{s \in(-\infty, 0]} \sum_{i=1}^{n}\left|\phi_{i}(s)-\psi_{i}(s)\right|$. Let $M=M_{0} / m_{0}$, then

$$
\|u(t, \phi)-u(t, \psi)\|_{1} \leq M\|\phi-\psi\|_{1} e^{-\varepsilon t}, \quad t>0 .
$$

We can always choose a positive integer $N$ such that $e^{-\varepsilon N \omega} M \leq 1 / 2$ and define a Poincaré mapping $P: C \rightarrow C$ by $P(\phi)=u_{\omega}(\phi)$. It follows from (34) that

$$
\left\|P^{N} \phi-P^{N} \psi\right\|_{1} \leq \frac{1}{2}\|\phi-\psi\|_{1},
$$

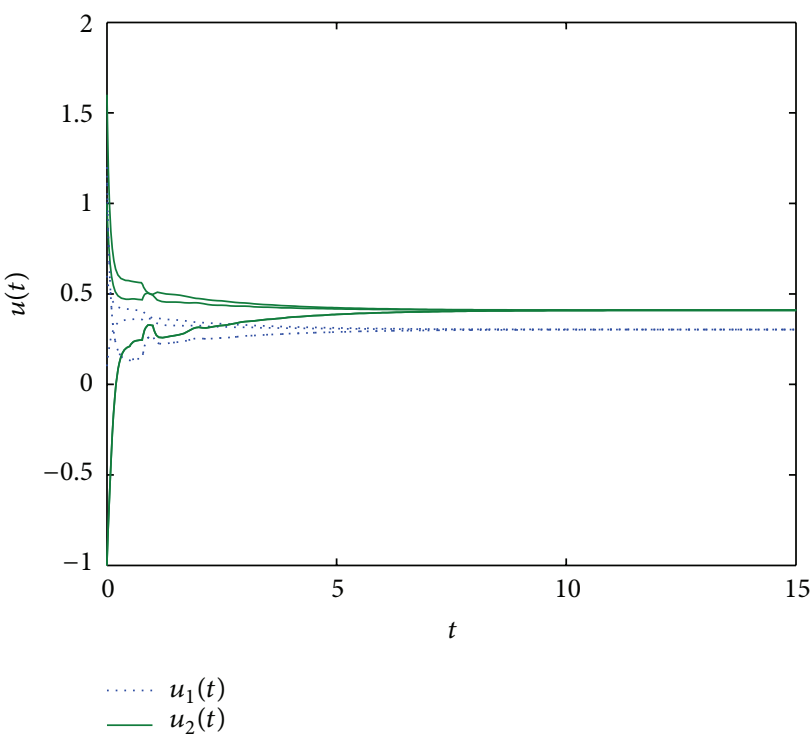

Figure 1: Time responses of the state variables $u(t)$ with different initial values in Example 1.

which implies that $P^{N}$ is a contraction mapping. Thus, there exists a unique fixed point $\varphi^{*}$ such that $P^{N} \varphi^{*}=\varphi^{*}$. Note that $P^{N}\left(P \varphi^{*}\right)=P\left(P^{N} \varphi^{*}\right)=P \varphi^{*}$. It means that $P \varphi^{*}$ is also a fixed point of $P^{N}$, then $P \varphi^{*}=\varphi^{*}$; that is, $u_{\omega}\left(\varphi^{*}\right)=\varphi^{*}$. Obviously, if $u\left(t, \varphi^{*}\right)$ is the solution of (2) through $\left(0, \varphi^{*}\right), u\left(t+\omega, \varphi^{*}\right)$ is also a solution of (2) and $u_{t+\omega}\left(\varphi^{*}\right)=u_{t}\left(u_{\omega}\left(\varphi^{*}\right)\right)=$ $u_{t}\left(\varphi^{*}\right)$ for $t>0$. This shows that $u\left(t, \varphi^{*}\right)$ is exactly an $\omega$ periodic solution of system (2) and all other solutions of (2) exponentially converge to it as $t \rightarrow+\infty$. This completes the proof.

Remark 14. The periodic oscillatory behavior of the neural networks is of great interest in many applications. For instance, this phenomena of periodic solutions for neural networks coincide with the fact that learning usually requires repetition and periodic sequences of neural impulse are also of fundament significance for the control of dynamic functions of the body such as heart beat and respiration which occur with great regularity.

Remark 15. In [23], the authors studied the existence and attractivity of periodic solutions for two class of CGNNs with discrete time delays or finite distributed time delays, respectively. In this paper, we incorporated time-varying delays and infinite distributed delays into CGNNs and derived the uniqueness and global exponential stability of periodic solutions. In [24, 27], two classes of CGNNs with distributed delays were investigated, and sufficient conditions were established to guarantee the uniqueness and global exponential stability of periodic solutions of such networks by using Lyapunov functional and the properties of $M$-matrix, whereas, the time-varying delays were ignored in the models. Thus, our results effectually improve or complement the results in $[23,24,27]$. 

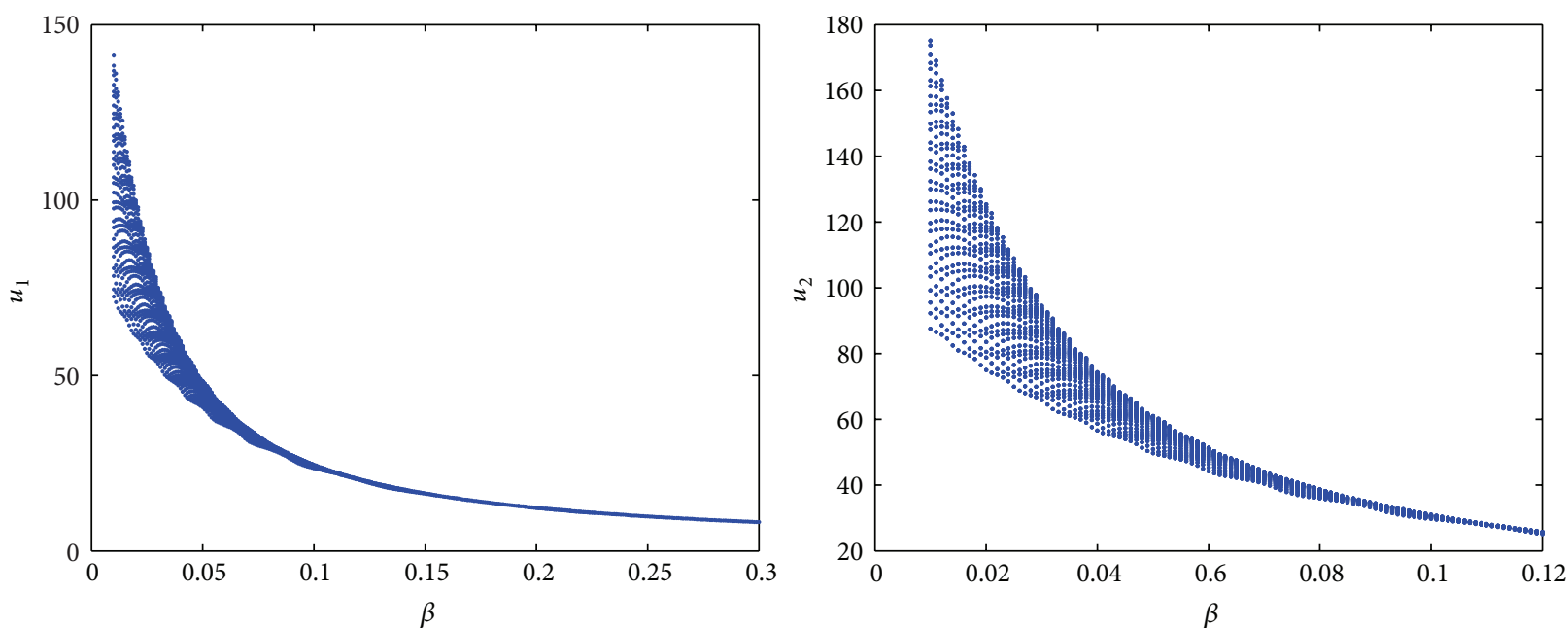

Figure 2: Bifurcation diagrams of system (2), and these show the effect of the parameter $\beta$ on the dynamic behavior.
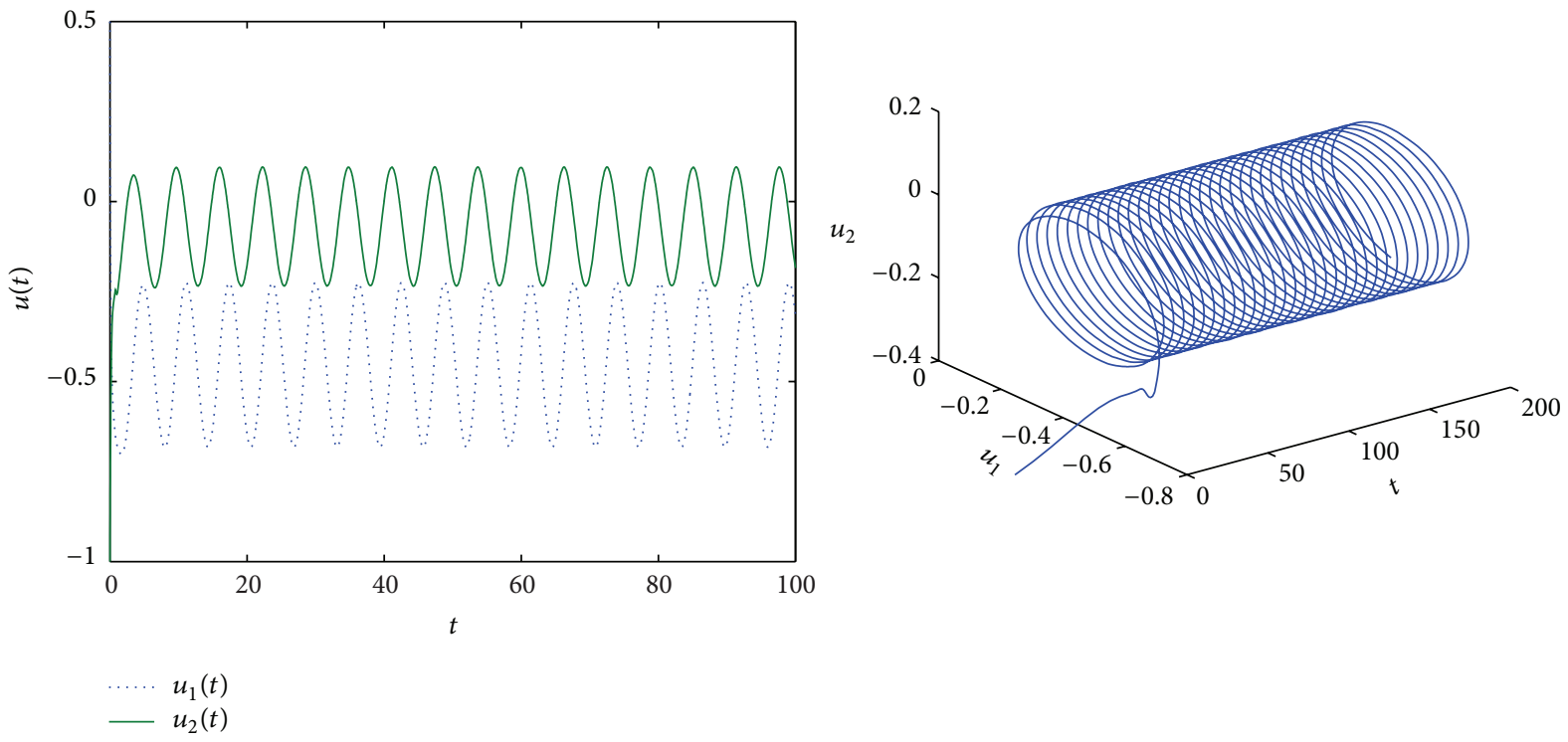

FIGURE 3: Time responses of the state variables $u(t)$ and phase plot in space $\left(t, u_{1}, u_{2}\right)$.

\section{Numerical Simulation}

In what follows, we give two examples to illustrate the results obtained in Sections 3 and 4.

Example 1. In system (2), we choose

$$
\begin{array}{ll}
\underline{A}=\left(\begin{array}{ll}
-0.3 & -0.2 \\
-0.5 & -0.6
\end{array}\right), & \bar{A}=\left(\begin{array}{cc}
0.3 & 0.2 \\
0.2 & 0.1
\end{array}\right), \\
\underline{B}=\left(\begin{array}{cc}
-0.8 & -0.9 \\
-0.4 & -1
\end{array}\right), & \bar{B}=\left(\begin{array}{cc}
0.5 & 0.6 \\
0.7 & 1
\end{array}\right), \\
\underline{C}=\left(\begin{array}{ll}
-0.5 & -0.5 \\
-0.3 & -0.8
\end{array}\right), & \bar{C}=\left(\begin{array}{cc}
0.5 & 0.6 \\
0.3 & 1
\end{array}\right),
\end{array}
$$

$$
\begin{gathered}
\widetilde{\alpha}_{i}\left(u_{i}(t)\right)=2+\sin \left(u_{i}(t)\right), \quad \widetilde{\beta}_{i}\left(u_{i}(t)\right)=5 u_{i}(t), \\
J_{1}=-1, \quad J_{2}=-1.5, \quad f_{j}(x)=\tanh (x), \\
\tau_{j}(t)=1-\frac{e^{-t}}{2}, \quad k_{j}(t)=t e^{-t}, \quad i, j=1,2 .
\end{gathered}
$$

It is clear that $\gamma_{1}=\gamma_{2}=5, l_{1}=l_{2}=1, \tau=1, \delta=0.5$, $\mu=1$. Using the optimization toolbox of Matlab and solving the optimization problem (10), we obtain

$$
\begin{gathered}
p_{1}=1.6495, \quad p_{2}=1.4828, \quad q_{1}=2, \\
q_{2}=2, \quad r_{1}=1.2143, \quad r_{2}=2 .
\end{gathered}
$$


By Theorem 9, system (2) is globally robustly exponentially stable. To illustrate the theoretical result, we present a simulation with

$$
\begin{gathered}
A=\left(\begin{array}{cc}
0.2 & 0.1 \\
-0.1 & -0.4
\end{array}\right), \quad B=\left(\begin{array}{ll}
0.1 & 0.5 \\
0.6 & 0.5
\end{array}\right), \\
C=\left(\begin{array}{cc}
0.2 & 0.36 \\
0.2 & 0.8
\end{array}\right) .
\end{gathered}
$$

We can find that the neuron vector $u(t)=\left(u_{1}(t), u_{2}(t)\right)^{T}$ converges to the unique equilibrium point $x^{*}=$ $(0.3031,0.4095)^{T}$ (see Figure 1$)$.

For system (2), we choose $\widetilde{\beta}_{i}\left(u_{i}(t)\right)=\beta u_{i}(t)$. In Figure 2, we exhibit a typical bifurcation and chaos diagrams when we fix other parameters as (36) and (38) and choose $\beta$ as a bifurcation parameter $(0.01 \leq \beta \leq 0.3)$. It clearly shows that system (2) admits rich dynamics including period-doubling bifurcation and chaos.

Example 2. In system (2), we take $\tau_{1}(t)=\tau_{2}(t)=1, J_{1}(t)=$ $2+\sin t, J_{2}(t)=\cos t$, and the other parameters are the same as those in (36). One can obtain that $\mathscr{M}=\left(\begin{array}{cc}3.6 & -2.6 \\ -2.2 & 2.4\end{array}\right)$, which is a nonsingular $M$-matrix. According to Theorem 13, system (2) has a $2 \pi$-periodic solution which is globally exponentially stable. We present a simulation with the parameters in (38) (see Figure 3).

\section{Conclusion}

In this paper, we discussed a class of interval CGNNs with time-varying delays and infinite distributed delays. By employing $H$-matrix and $M$-matrix theory, Lyapunov functional method, and LMI approach, sufficient conditions were established for the existence, uniqueness, and global robust exponential stability of the equilibrium point and the periodic solution to the neural networks. It was shown that the obtained results improve or complement the previously published results. Numerical simulations demonstrated the main results and further showed that chaotic phenomena may occur for the system, which coincide with the fact of recognition character of human beings. On the other hand, it is well known that chaotic synchronization has been successfully applied to secure communication; chaotic behaviors of neural networks imply that they may be used to create secure communication systems.

\section{Acknowledgment}

This work was supported by the National Natural Science Foundation of China (11071254).

\section{References}

[1] W. Han, Y. Liu, and L. Wang, "Robust exponential stability of Markovian jumping neural networks with mode-dependent delay," Communications in Nonlinear Science and Numerical Simulation, vol. 15, no. 9, pp. 2529-2535, 2010.
[2] O. M. Kwon, S. M. Lee, and J. H. Park, "Improved delay-dependent exponential stability for uncertain stochastic neural networks with time-varying delays," Physics Letters A, vol. 374, no. 10, pp. 1232-1241, 2010.

[3] X. Li, "Global robust stability for stochastic interval neural networks with continuously distributed delays of neutral type," Applied Mathematics and Computation, vol. 215, no. 12, pp. 4370-4384, 2010.

[4] J.-L. Shao, T.-Z. Huang, and X.-P. Wang, "Improved global robust exponential stability criteria for interval neural networks with time-varying delays," Expert Systems with Applications, vol. 38, no. 12, pp. 15587-15593, 2011.

[5] J.-L. Shao, T.-Z. Huang, and S. Zhou, "An analysis on global robust exponential stability of neural networks with time-varying delays," Neurocomputing, vol. 72, no. 7-9, pp. 1993-1998, 2009.

[6] J.-L. Shao, T.-Z. Huang, and S. Zhou, "Some improved criteria for global robust exponential stability of neural networks with time-varying delays," Communications in Nonlinear Science and Numerical Simulation, vol. 15, no. 12, pp. 3782-3794, 2010.

[7] F. Wang and H. Wu, "Mean square exponential stability and periodic solutions of stochastic interval neural networks with mixed time delays," Neurocomputing, vol. 73 , no. 16-18, pp. 3256-3263, 2010.

[8] W. Zhao and Q. Zhu, "New results of global robust exponential stability of neural networks with delays," Nonlinear Analysis: Real World Applications, vol. 11, no. 2, pp. 1190-1197, 2010.

[9] H. Liu, Y. Oua, J. Hu et al., "Delay-dependent stability analysis for continuous-time BAM neural networks with Markovian jumping parameters," Neural Networks, vol. 23, pp. 315-321, 2010.

[10] J. Pan, X. Liu, and S. Zhong, "Stability criteria for impulsive reaction-diffusion Cohen-Grossberg neural networks with time-varying delays," Mathematical and Computer Modelling, vol. 51, no. 9-10, pp. 1037-1050, 2010.

[11] J. Tian and S. Zhong, "Improved delay-dependent stability criterion for neural networks with time-varying delay," Applied Mathematics and Computation, vol. 217, no. 24, pp. 10278-10288, 2011.

[12] H. Wang, Q. Song, and C. Duan, "LMI criteria on exponential stability of BAM neural networks with both time-varying delays and general activation functions," Mathematics and Computers in Simulation, vol. 81, no. 4, pp. 837-850, 2010.

[13] X. Zhang, S. Wu, and K. Li, "Delay-dependent exponential stability for impulsive Cohen-Grossberg neural networks with time-varying delays and reaction-diffusion terms," Communications in Nonlinear Science and Numerical Simulation, vol. 16, no. 3, pp. 1524-1532, 2011.

[14] X. Fu and X. Li, "LMI conditions for stability of impulsive stochastic Cohen-Grossberg neural networks with mixed delays," Communications in Nonlinear Science and Numerical Simulation, vol. 16, no. 1, pp. 435-454, 2011.

[15] K. Li, "Stability analysis for impulsive Cohen-Grossberg neural networks with time-varying delays and distributed delays," Nonlinear Analysis: Real World Applications, vol. 10, no. 5, pp. 2784-2798, 2009.

[16] B. Zhou, Q. Song, and H. Wang, "Global exponential stability of neural networks with discrete and distributed delays and general activation functions on time scales," Neurocomputing, vol. 74, no. 17, pp. 3142-3150, 2011.

[17] P. Balasubramaniam and M. S. Ali, "Robust exponential stability of uncertain fuzzy Cohen-Grossberg neural networks with 
time-varying delays," Fuzzy Sets and Systems, vol. 161, no. 4, pp. 608-618, 2010.

[18] W. Su and Y. Chen, "Global robust stability criteria of stochastic Cohen-Grossberg neural networks with discrete and distributed time-varying delays," Communications in Nonlinear Science and Numerical Simulation, vol. 14, no. 2, pp. 520-528, 2009.

[19] Z. Wang, H. Zhang, and W. Yu, "Robust stability criteria for interval Cohen-Grossberg neural networks with time varying delay," Neurocomputing, vol. 72, no. 4-6, pp. 1105-1110, 2009.

[20] Z. Huang and Y. Xia, "Exponential periodic attractor of impulsive BAM networks with finite distributed delays," Chaos, Solitons \& Fractals, vol. 39, no. 1, pp. 373-384, 2009.

[21] S. Townley, A. Ilchmann, M. G. Weiss et al., "Existence and learning of oscillations in recurrent neural networks," IEEE Transactions on Neural Networks, vol. 11, no. 1, pp. 205-214, 2000.

[22] X. Chen and Q. Song, "Global exponential stability of the periodic solution of delayed Cohen-Grossberg neural networks with discontinuous activations," Neurocomputing, vol. 73, no. 16-18, pp. 3097-3104, 2010.

[23] C.-H. Li and S.-Y. Yang, "Existence and attractivity of periodic solutions to non-autonomous Cohen-Grossberg neural networks with time delays," Chaos, Solitons and Fractals, vol. 41, no. 3, pp. 1235-1244, 2009.

[24] Q. Liu and R. Xu, "Periodic solutions of high-order Cohen-Grossberg neural networks with distributed delays," Communications in Nonlinear Science and Numerical Simulation, vol. 16, no. 7, pp. 2887-2893, 2011.

[25] J. Pan and Y. Zhan, "On periodic solutions to a class of nonautonomously delayed reaction-diffusion neural networks," Communications in Nonlinear Science and Numerical Simulation, vol. 16, no. 1, pp. 414-422, 2011.

[26] H. Xiang and J. Cao, "Exponential stability of periodic solution to Cohen-Grossberg-type BAM networks with time-varying delays," Neurocomputing, vol. 72, no. 7-9, pp. 1702-1711, 2009.

[27] Q. Liu and R. Xu, "Periodic solutions of a Cohen-Grossbergtype BAM neural networks with distributed delays and impulses," Journal of Applied Mathematics, vol. 2012, Article ID 643418, 17 pages, 2012.

[28] J. C. Principe, J.-M. Kuo, and S. Celebi, "An analysis of the gamma memory in dynamics neural networks," IEEE Transactions on Neural Networks, vol. 5, no. 2, pp. 331-337, 1994.

[29] J. Zhang, "Global exponential stability of interval neural networks with variable delays," Applied Mathematics Letters, vol. 19, no. 11, pp. 1222-1227, 2006.

[30] R. A. Horn and C. R. Johnson, Topics in Matrix Analysis, Cambridge University Press, Cambridge, Mass, USA, 1991.

[31] H. Zhang, Z. Wang, and D. Liu, "Robust exponential stability of recurrent neural networks with multiple time-varying delays," IEEE Transactions on Circuits and Systems II, vol. 54, no. 8, pp. 730-734, 2007.

[32] Y. Du and R. Xu, "Global robust exponential stability analysis for interval neural networks with mixed delays," Abstract and Applied Analysis, vol. 2012, Article ID 647231, 18 pages, 2012.

[33] T. Ensari and S. Arik, "New results for robust stability of dynamical neural networks with discrete time delays," Expert Systems with Applications, vol. 37, no. 8, pp. 5925-5930, 2010.

[34] N. Ozcan and S. Arik, "Global robust stability analysis of neural networks with multiple time delays," IEEE Transactions on Circuits and Systems I, vol. 53, no. 1, pp. 166-176, 2006.
[35] V. Singh, "Improved global robust stability criterion for delayed neural networks," Chaos, Solitons and Fractals, vol. 31, no. 1, pp. 224-229, 2007. 


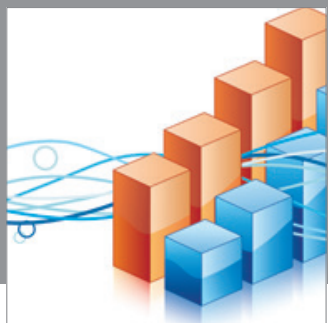

Advances in

Operations Research

mansans

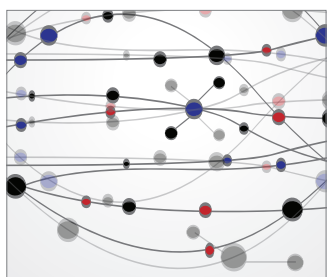

The Scientific World Journal
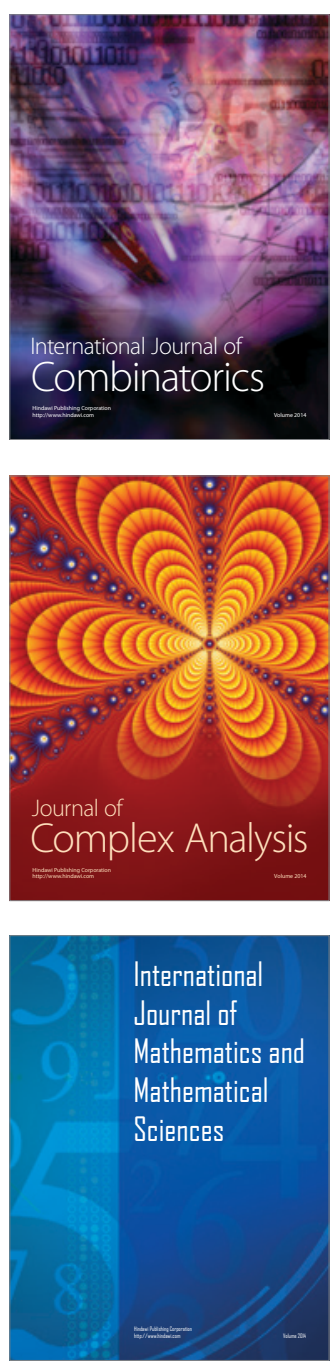
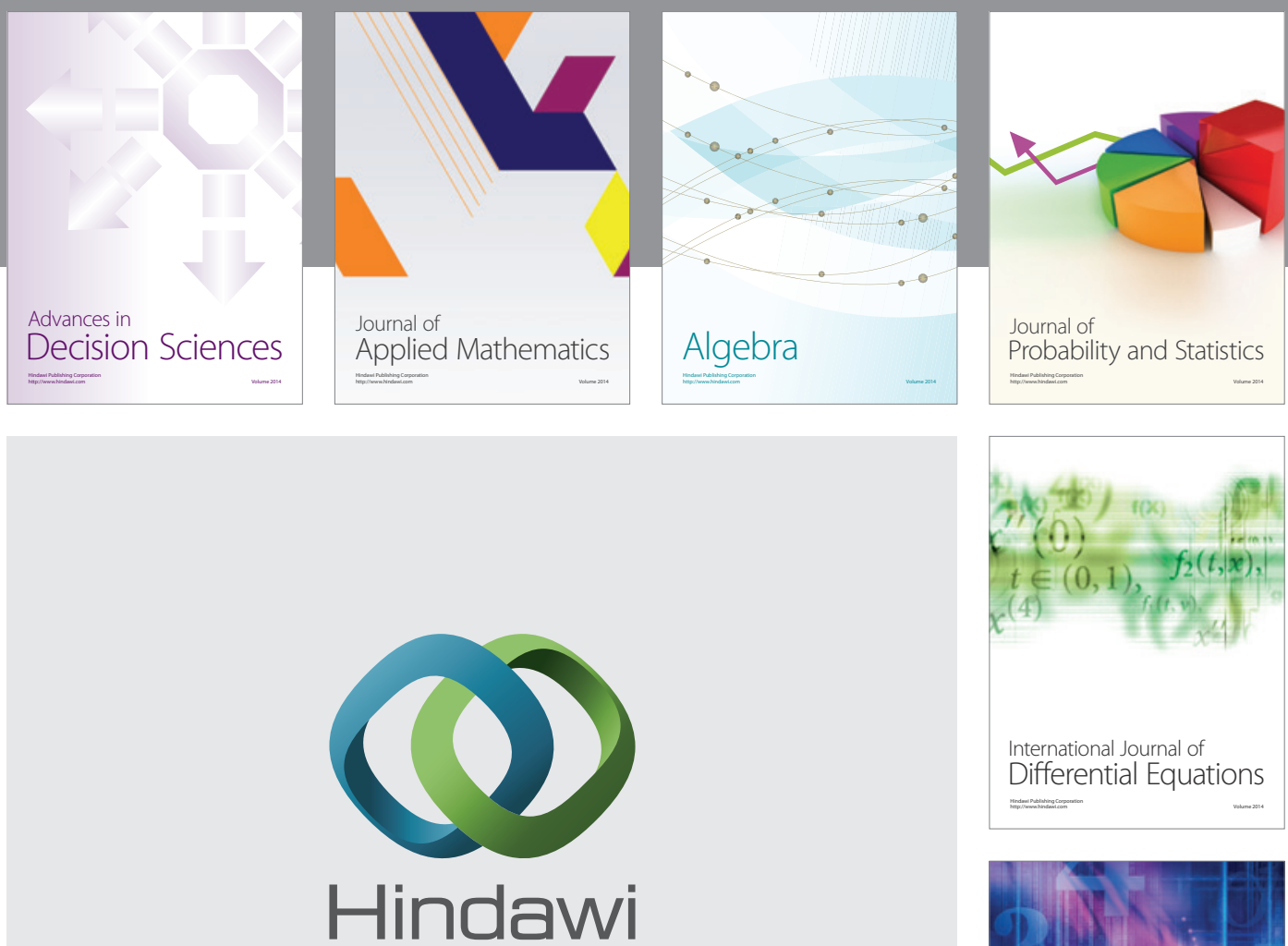

Submit your manuscripts at http://www.hindawi.com
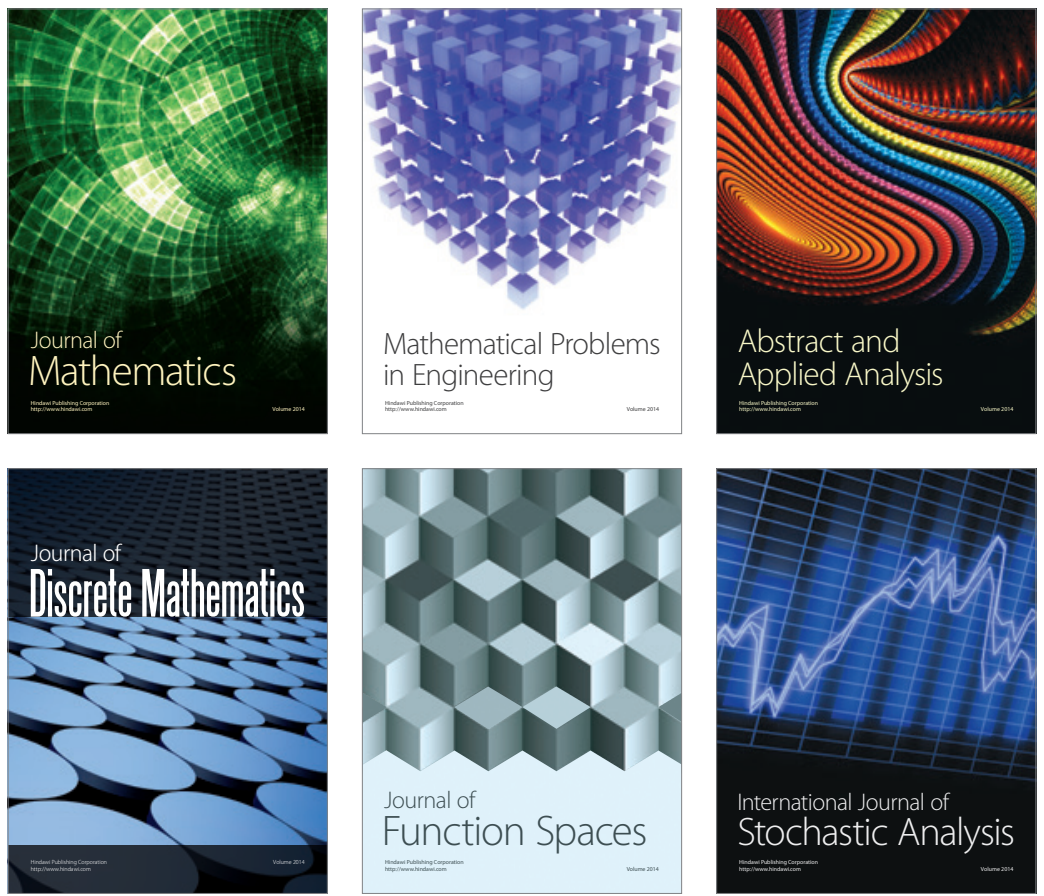

Journal of

Function Spaces

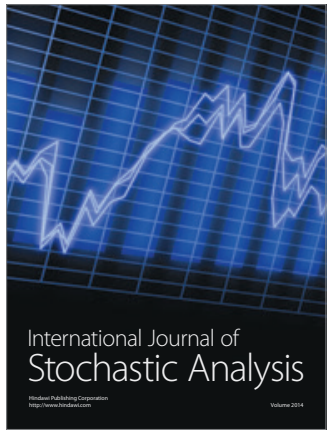

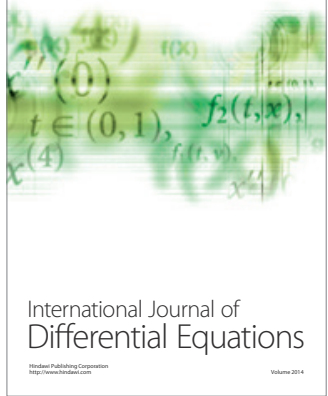
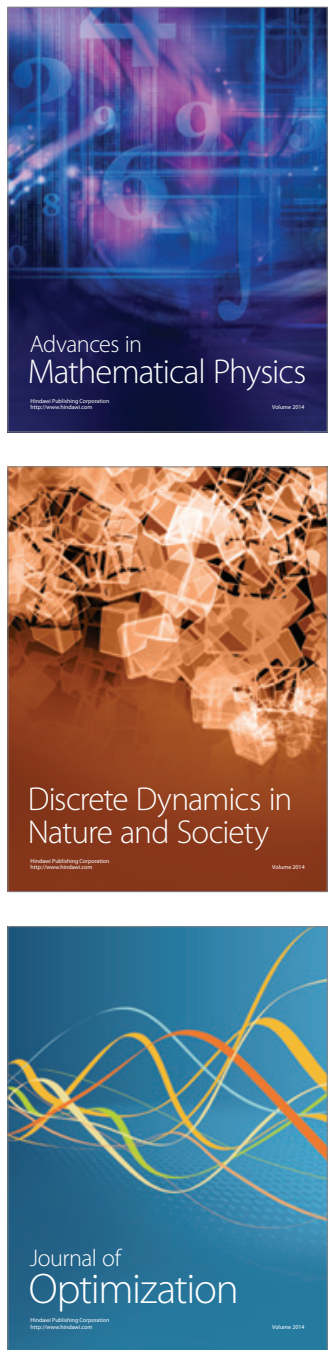